\title{
EFFECTS OF COGNITIVE AND PHYSICAL DECLINE ON OLDER DRIVERS'SIDE-TO- SIDE SCANNING FOR HAZARDS WHILE EXECUTING TURNS
}

\author{
Matthew Romoser and Donald L. Fisher \\ University of Massachusetts Amherst \\ Amherst, Massachusetts, USA \\ Email: mromoser@ecs.umas.edu, fisher@ecs.umass.edu
}

\begin{abstract}
Summary: Age related declines in cognitive and physical ability significantly impair an older adult's ability to safely drive. As we age it gradually becomes more difficult to scan for, detect, process, and ultimately react to critical elements in our driving environment. Older drivers are over represented in angled impacts in intersections. Research has shown that older drivers tend to execute fewer side-to-side glances while in the process of turning than middle-aged drivers. This decrease in scanning can directly lead to an increase in angled impacts. The present research investigates the correlation between cognitive and physical decline and the likelihood that an older driver will execute side-to-side glances at the beginning and during a turn. Results of both simulator and field drive sessions with fifty-four older drivers 70-89 years of age demonstrated that cognitive, but not physical, decline was significantly correlated with a decrease in side-to-side scanning while turning.
\end{abstract}

\section{INTRODUCTION}

In order to navigate safely on the road, drivers must be able to perceive information in their environment, process and assimilate that information into an accurate mental picture or map, and then execute appropriate responses to their situation. Failure to do so efficiently and accurately can lead to a crash. Unfortunately, age related declines in visual, cognitive, and psychomotor ability all impact one's ability to do these things. Drivers over the age of seventy have a higher proportion of accidents per vehicle mile traveled (VMT) than younger, experienced drivers. Drivers over the age of eighty have a crash rate that is approximately two-and-a-half to three times that of drivers between the ages of thirty to sixty-four (Bayam, Liebowitz \& Agresti, 2005; Bryer, 2000; Ryan, Legge \& Rosman, 1998). When this data is parsed into crash type, it is revealed that the rate of angled collisions increases significantly after age seventy while the rates of all other crash types, including rear-end and head-on crashes, decreases with age. The majority of angled collisions involving drivers over the age of seventy occur in intersections, the highest percentage of which are during left turns (Bryer, 2000).

Older adults have a diminished capacity for processing information compared to younger adults, especially in the periphery. This is due in large part to an age related decline in short-term memory capacity and speed-of-processing (Ball, 1997; Bolstad \& Hess, 2000; Cerella, 1990). The speed at which information is processed plays an important role in keeping drivers out of crashes. Information about crash risks must be incorporated into a driver's situational assessment, maintained when not attended to, and then reacted upon quickly. Most driving environments contain a large amount of dynamically changing information. With each passing second, new information presents itself that must be incorporated into the driver's situational 
assessment in order to safely navigate the many potential hazards that can exist. Shinar, Mcdonald \& Treat (1978) estimate that 25\% to 50\% of traffic accidents occur as a result of inadequate monitoring of the driving environment. For drivers over the age of sixty, crash risk is positively correlated with information processing time (Eby et al., 1998; Klavora \& Heslegrave, 2002). Reaction time to the appearance of stimuli also decreases with age (Eby et al., 1998; Glass et al., 2000).

Age related declines in physical and psychomotor coordination could also affect a person's ability to drive a car. With advancing age, the neck and torso can become more stiff and inflexible. Coordination and speed of movements with the arms, hands and head also declines (McGill, Yingling \& Peach, 1999). As one ages, it is normal to experience a decrease in the range of motion of the joints, tendons, and muscles of the body (Malfetti, 1985). Those particularly important to driving, mobility of the neck and torso, are no exception (Eby et al., 1998; Janke, 1994). It has been shown that reduced flexibility of the neck and torso contributes to an increased likelihood of a crash while driving (McPherson, Michael, Ostrow, \& Shaffron, 1988; McPherson, Ostrow, Shaffron, \& Yeater, 1989). Isler, Parsonson, \& Hansson (1997) found that the increased inability to move one's head significantly reduced the distance at which oncoming vehicles could be perceived. Older drivers are less effective at scanning the road for information than younger drivers. Measurable age-related differences exist in road scanning behavior (Romoser, Fisher, Mourant, Wachtel \& Sizov, 2005; Underwood, Phelps, Wright, van Loon), saccadic eye movements and motion perception (Schieber, 2006). In each case, older drivers are not as effective doing these things as younger drivers.

The current study investigated the relationship between cognitive and physical decline and the likelihood of executing secondary sideways glances after beginning a turn both in a simulator and in the field. In a previous simulator study by Romoser, et al. (2005), it was found that, after having begun rolling into the intersection to make a turn to either the right or left, older drivers failed to look to the side to monitor for new traffic in the intersection three times more often than younger experienced drivers. While scanning side-to-side while stopped prior to entering an intersection is important, continued side-to-side scanning just after beginning a turn is critical for early detection of new, previously hidden or unseen vehicles. Cognitive slowing results in the ability to detect, process and react to information in the driving environment. Age related physical declines make it more difficult and increases the time required to physically scan the driving environment. Together, these can lead to a decrease in a driver's situational awareness, which in turn can result in a crash (Endsley, 1995).

\section{METHOD}

For the current study, there were a total of three sessions with each participant spread out over several weeks. In the first session a battery of cognitive, psychomotor and physical tests were administered. The second session consisted of a series of simulated drives targeted at evaluating the participant's side-to-side scanning while making turns. The third session consisted of a field drive that began and ended at the participant's home. 


\section{Participants}

Participants were fifty-four older drivers between the ages of seventy and eighty-nine. Stratified random sampling was used to ensure age was evenly distributed. There were eighteen participants in each of three age groups: seventy to seventy-four, seventy-five to seventy-nine and eighty to eighty-nine. Gender was balanced within each age group with nine males and nine females. Participants were recruited through a direct letter writing campaign and by posting flyers in seven towns in and around Amherst, Massachusetts. To be eligible for the study, participants had to have a valid U.S. driver's license and have been driving for at least ten years without any restrictions on their license.

\section{Session One: Physical \& Cognitive Testing}

Session one was the longest session and lasted approximately two hours. During session one participants were administered a series of cognitive, physical and psychomotor tests. The cognitive tests used were the Rey Auditory Verbal Learning Test (20 min recall) and the Complex Figure Test. The physical tests used were Snellen Far and Near visual acuity, sitting flexibility (left and right), and the Get Up and Go test. The psychomotor tests used were the Trail Making Test 2 and the Grooved Pegboard test. Screening tests took approximately an hour and a half to administer. After a short break, participants were given the opportunity to practice driving the simulator in order to become familiar with driving in a virtual environment and orient themselves with the control of the simulator. If a participant experienced symptoms of simulator sickness, they were excused from further participation and were replaced in the study by a participant fitting the same age and gender demographic. Attrition due to simulator sickness was $38.6 \%$.

\section{Session Two: Simulator Drives}

During session two, which lasted approximately one hour, participants drove a series of four separate simulator runs with three to four minute breaks between each. Each run was five to seven minutes in length. The first drive was a repeat of the practice drive from session one to allow the participant an opportunity to reorient to the experience of driving the simulator. The remaining three simulator drives were evaluation runs.

The driving simulator used was the Advanced Driving Simulator at the Human Performance Lab at the University of Massachusetts Amherst. The simulator consists of a full-body 1995 Saturn sedan surrounded by three projection screens. The virtual roadway is projected upon these three screens and covers 135 degrees of visual angle from the driver's point-of-view. The system provides realistic road, wind and other vehicle noises with appropriate direction and intensity. The lab's four-camera mobile lab system (Figure 1) was used to record the participant's drive for later analysis. The system consists of a small wireless color camera that was worn by the participant on a lightweight headband and three environmental cameras. The headband camera's purpose was to record large, side-to-side head movements during turns. The remaining three cameras were placed on top of the simulator and recorded the center, left and right screens. The four video signals were then multiplexed and recorded on a portable digital videocassette 
recorder for later analysis. This mobile setup was also used during the field drive in session three.

After introductions and instructions were completed, the participant was fitted with the wireless headband camera and seated in the simulator. After the practice drive, the participant was asked to drive the three trial blocks that contained the ten intersections with peripheral hazards. In these intersections, participants performed actions such as turning left and merging with traffic, turning right and merging with traffic or turning left across traffic. In each case there was an area near the intersection to the side of the driver's vehicle that could potentially conceal a moving vehicle or pedestrian. An example of one of the scenarios used can be found in Figure 2.

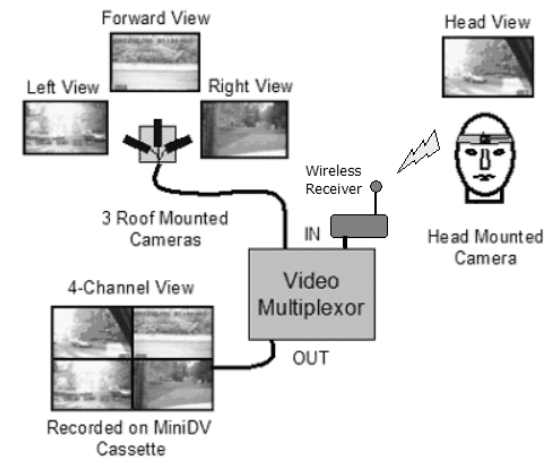

Figure 1. Four camera mobile lab system.

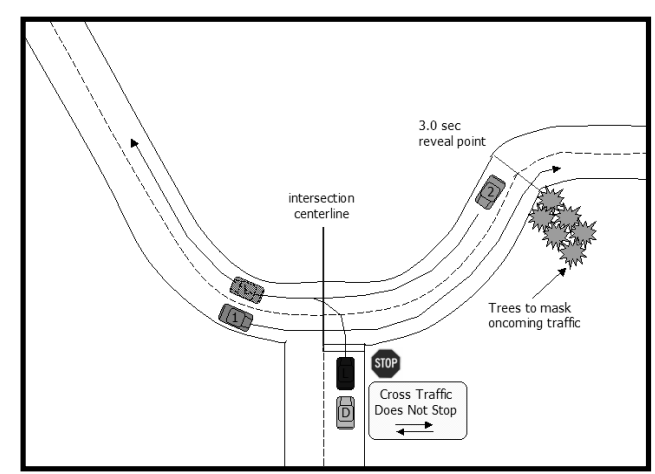

Figure 2. Example of intersection with hazard. Participant (D) makes left turn following lead vehicle $(L)$ at $T$-intersection with blind curve to the right (reveal point) three seconds away from intersection concealing car (2).

Videos of the drives were recorded and later analyzed via an unbiased blind review. The type of scanning performed by the driver (a) while preparing for the turn and then (b) during the turn was evaluated. Side-to-side glances while approaching or stopped at the intersection (primary glances) were not counted. Continued side-to-side scanning early in the turn just after the driver began to roll into the intersection (secondary glance) was scored. The performance criterion used for analysis was whether or not the participant took a secondary glance towards the area of the peripheral hazard after having begun their turn for each of the ten simulated intersections. Secondary glances during a turn, especially early in the turn just as the vehicle crosses into the threshold of the intersection, are critical for maintaining situational awareness and making it more likely a driver reacts in time to avoid a collision with unanticipated vehicles approaching the intersection.

\section{Session Three: Field Drives}

Approximately two thirds of the older drivers in the study also participated in a field drive. For safety reasons and because field drives of this kind had not been done before, participation was limited to drivers between the ages of seventy and seventy-nine. The objective of the field drive was to capture naturalistic driving behavior in an environment and vehicle with which the older driver was comfortable. To accomplish this goal, the field drive began and ended at the 
participant's home. At the beginning of the session, the experiment administrator met the participant at his or her home and then installed the four-camera mobile lab system (see Figure 1) in the participant's own vehicle. The participant and administrator then agreed on a route that would require approximately thirty minutes to drive, contained several left and right turns, and ended back at the participant's home. Once the route was chosen, the participant was fitted with the wireless headband scene camera. The participant then climbed into his or her vehicle, recording of the drive was started, and the drive began. The administrator did not accompany the participant on the field drive. Again, this was done to minimize anxiety and reduce feelings of the administrator "watching my every move".

The above steps were taken to increase the participants' overall comfort with the experience of being evaluated and decrease the study's overall risk. We believed that having the participants travel to campus only to have them drive an unfamiliar test vehicle on unfamiliar roads would unnecessarily increase anxiety, make it less likely that we would capture naturalistic driving behavior, and increase the likelihood of a crash because of the increased workload these factors would introduce. To our knowledge, there is no other driving field study in the literature that has used such a procedure. For session three, secondary glances during turns in intersections were later evaluated via blind review in the same manner as they were for session two.

\section{RESULTS}

An analysis was undertaken of the extent to which cognitive and physical status could be used to predict whether or not participants would execute secondary looks when beginning their turns. To eliminate the issue of collinearity, the results of the cognitive and psychomotor measures were collapsed into a single "Cognitive Status" variable. The results of the physical measures were collapsed into a single "Physical Status" variable. Each test score was given equal weight. To determine the score for the Cognitive and Physical Status variables, the participant's normalized Z-score on each measure relative to the mean and standard deviation of all participants on that measure was determined. The Z-scores were then summed to arrive at an overall Cognitive (cognitive \& psychomotor measures) and Physical Status (physical measures) score.
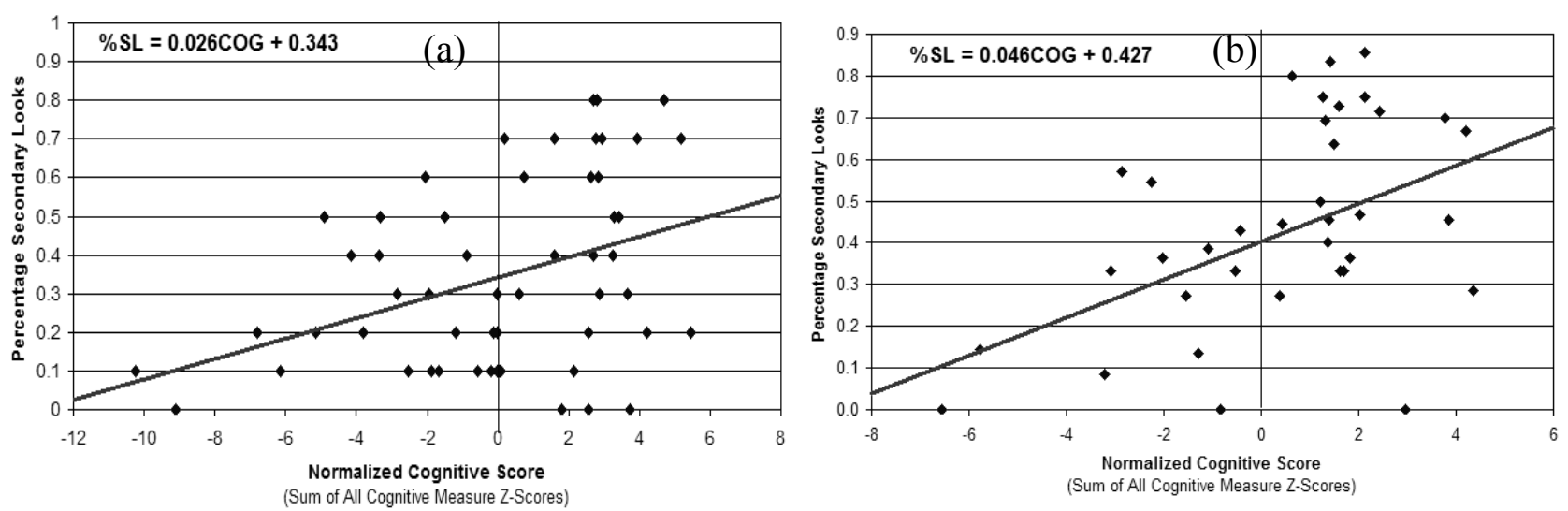

Figure 3. Scatterplot of cognitive status versus percentage of secondary looks during (a) simulator drive (54 participants) and (b) field drive (36 participants). Note that, for safety reasons, participation in the field drive was limited to those seventy to seventy-nine years of age. 
For the simulator drive data, Cognitive Status was found to be a significant predictor of the likelihood of secondary looks $(t=2.919, R=.375, p<0.01)$. Physical Status was not a significant predictor and was subsequently removed from the regression model. Similar results were found for the field drive data. For the field drives, Cognitive Status was again found to be a significant predictor of the likelihood of secondary looks $(t=3.240, R=.486, p<0.005)$. Again, Physical Status was not a significant predictor and was removed from the model. Simulator and field drive regression results are summarized in Figure 3.

Finally, there was a statistically significant positive correlation between secondary looks during simulator drives and field drives $\left(r=0.411, R^{2}=0.17, p<0.05\right)$. In general, this can be interpreted to mean that participants who did not reliably execute secondary looks in the simulator tended to also not execute secondary looks while driving their own cars during the field drive. Conversely, drivers who did reliably take secondary looks in the simulator tended to also execute them in the field.

\section{DISCUSSION}

Older drivers are over represented in angled impacts in intersections. Previous simulator research has shown that older drivers tend to turn their heads less frequently side-to-side while executing turns than younger, middle-aged drivers (Romoser et al., 2005). The primary contribution of the current research is that cognitive, but not physical, status was a significant predictor of the likelihood of secondary looks both in the simulator and in the field. The fact that cognitive ability and not physical status was a significant predictor of head turns is surprising. It may be an indication that the problem is more a function of cognitive workload than ability to physically turn one's head in the direction of the oncoming traffic. The implication is that age related cognitive decline might lead to decreases in secondary looks and a subsequent decrease in situational awareness, especially during turns. The act of taking a secondary look suggests a higher level of situational awareness - namely, the ability to predict the potential future state of the environment (Endsley, 1995).

Just as there is evidence that lower levels of situation awareness among older adults can be trained, in particular speed-of-processing (Roenker, Cissell, Ball, Wadley, \& Edwards, 2003), so recently it has been reported that higher levels of situation awareness and, in particular, increases in secondary looks among older drivers, can be trained (Romoser, 2008). Such training must necessarily complement speed-of-processing training since speed-of-processing training assumes a threat is within the driver's field of view. Training secondary looks is necessary as a first step in order to bring a threat into a driver's field of view.

\section{REFERENCES}

Ball, K. (1997). Attentional problems and older drivers. Alzheimer Disease and Associated Disorders, 11, 42-47.

Bayam, E., Liebowitz, J., \& Agresti, W. (2005). Older drivers and accidents: A meta analysis and data mining application on traffic accident data. Expert Systems with Applications, 29, 598-629. 
Bolstad, C. A. \& Hess, T. M. (2000). Situation awareness and aging. In M.R.Endile \& D. J. Garland (Eds.), Situation Awareness Analysis \& Measurement (pp. 277-302). Mahway, N.J.: Lawerence Erlbaum Associates.

Bryer, T. (2000). Characteristics of motor vehicle crashes related to aging. In K. W. Schaie \& M. Pietrucha (Eds.), Mobility and Transportation in the Elderly, 157-206. New York, NY: Springer Publishing Company, Inc.

Cerella, J. (1990). Aging and information-processing rate. In J.E.Birren \& K. W. Schaie (Eds.), Handbook of Psychology and Aging (3rd ed., pp. 202-221). San Diego, CA: Academic Press.

Eby, D. W., Trombley, D. A., Molnar, L. J., \& Shope, J. T. (1998). The Assessment of Older Drivers' Capabilities: A Review of the Literature (Rep. No. UMTRI 98-24). The University of Michigan, Transportation Research Institute.

Endsley, M. R. (1995). Toward a theory of situation awareness in dynamic systems. Human Factors 37(1), 32-64.

Glass, J. M., Schumacher, E. H., Lauber, E. J., Zurbriggen, E. L., et al. (2000). Aging and the psychological refractory period: Task-coordination strategies in young and old adults. Psychology and Aging, 15, 571-595.

Isler, R. B., Parsonson, B. S., \& Hansson, G. J. (1997). Age related effects of restricted head movements on the useful field of view of drivers. Accident Analysis and Prevention, 29, 793801.

Janke, M. K. (1994). Age-related disabilities that may impair driving and their assessment. Sacramento: California Department of Motor Vehicles (Report No. 156).

Klavora, P. \& Heslegrave, R. J. (2002). Senior drivers: An overview of problems and intervention strategies. Journal of Aging and Physical Activity, 10, 322-335.

Malfetti, J.W. (1985). Needs and Problems of Older Drivers: Survey Results and Recommendations. Falls Church, VA: AAA Foundation for Traffic Safety.

McGill, S. M., Yingling, V. R., \& Peach, J. P. (1999). Three-dimensional kinematics and trunk muscle myoelectric activity in the elderly spine - a database compared to young people. Clinical Biomechanics, 14, 389-395.

McPherson, K., Michael, J., Ostrow, A., \& Shaffron P. (1988). Physical Fitness and the Aging Driver. Phase I. Washington, DC: AAA Foundation for Traffic Safety.

McPherson, K., Ostrow, A., Shaffron, P., \& Yeater, R. (1989). Physical Fitness and the Aging Driver. Phase II. Washington, DC: AAA Foundation for Traffic Safety.

Roenker, D. L., Cissell, G. M., Ball, K., Wadley, V. G., \& Edwards, J. D. (2003). Speed-ofprocessing and driving simulator training result in improved driving performance. Human Factors, 45, 218-233.

Romoser, M., Fisher, D., Mourant, R., Wachtel, J., Sizov, K. (2005). The use of a driving simulator to assess senior driver performance: Increasing situational awareness through postdrive one-on-one advisement. 3rd International Driving Symposium on Human Factors in Driver Assessment, Training, and Vehicle Design, June 2005, Rockport, Maine, 456-463. 
Romoser, M. (2008). Improving the road scanning behavior of older drivers through the use of situation-based learning strategies. Doctoral Dissertation, University of Massachusetts Amherst, Department of Mechanical \& Industrial Engineering, 2008.

Ryan, G. A., Legge, M., \& Rosman, D. (1998). Age related changes in driver's crash risk and crash type. Accident Analysis and Prevention, 30, 379-387.

Schieber, F. (2006). Vision and aging. In Birren, J. E., Schaie, K. W. (Eds.), Handbook of the Psychology of Aging, 129-161. Amsterdam: Academic Press.

Shinar, D., Mcdonald, S., Treat, J. (1978). Interaction Between Driver Mental and Physical Conditions and Errors Causing Traffic Accidents - Analytical Approach, Journal of Safety Research, 10(1), 16-23.

West, R.L., Crook, T.H., \& Barron, K.L. (1992). Everyday memory performance across the lifespan: Effects of age and noncognitive individual differences. Psychology and Aging, 7, 7282. 